\title{
MAHLER MEASURES GENERATE THE LARGEST POSSIBLE GROUPS
}

\author{
ARtūRAS DUBICKAS
}

\begin{abstract}
We prove that free additive and multiplicative groups generated by the set of all Mahler measures consist of all real algebraic integers and of all positive algebraic numbers, respectively. More precisely, we show that every positive algebraic number can be written as a quotient of two Mahler measures. It is also shown that the set of all Mahler measures is not an additive semigroup.
\end{abstract}

\section{Introduction}

Let $\alpha$ be an algebraic number of degree $d$ over $\mathbb{Q}$ with minimal polynomial

$$
a_{d} X^{d}+\cdots+a_{1} X+a_{0}=a_{d}\left(X-\alpha_{1}\right) \cdots\left(X-\alpha_{d}\right) \in \mathbb{Z}[X] .
$$

Its Mahler measure is defined by $M(\alpha)=a_{d} \prod_{j=1}^{d} \max \left\{1,\left|\alpha_{j}\right|\right\}$. It is well-known that, for every $\alpha \in \overline{\mathbb{Q}}, M(\alpha)$ is a real algebraic integer greater than or equal to 1 .

Let $\mathcal{M}$ be the set of all Mahler measures of algebraic numbers, and let $\mathcal{M}^{*}$ be a monoid under multiplication generated by $\mathcal{M}$. By the multiplicative property of Mahler measures $\mathcal{M}^{*}$ is the set of all Mahler measures of integer (not necessarily irreducible) polynomials. A thorough investigation of the sets $\mathcal{M}$ and $\mathcal{M}^{*}$ is considered to be a very ambitious task [2], since even the apparently simple question of Lehmer [8] on whether there are elements of $\mathcal{M}$ in the interval $(1,1.176)$ remains open.

The aim of this note is to show that the set $\mathcal{M}$ is very rich. Denote by $P$ the set of Perron numbers, i.e. positive algebraic numbers $\alpha$ such that the moduli of their conjugates (if any) are all strictly smaller than $\alpha$. Let $\overline{\mathbb{Q}}_{>0}$ be the multiplicative group of all positive algebraic numbers, and let $G=\left\{\prod m_{j}^{k_{j}} \mid m_{j} \in\right.$ $\left.\mathcal{M}, k_{j} \in \mathbb{Z}\right\}$ be a free multiplicative group generated by all Mahler measures. With this notation, we have that

$$
\mathcal{M} \subseteq \mathcal{M}^{*} \subseteq P \subseteq G \subseteq \overline{\mathbb{Q}}_{>0}
$$

It was shown in [4] that $\mathcal{M} \neq \mathcal{M}^{*}$ with, say $\alpha=(\sqrt{2}+1)(\sqrt{3}+2) \in \mathcal{M}^{*} \backslash \mathcal{M}$. Hence $\mathcal{M} \subset \mathcal{M}^{*}$. The fact that every Mahler measure (and so their product) is a Perron number was firstly noticed by Adler and Marcus [1]. Of course, not

Received July 4, 2003.

2000 AMS Subject Classification: 11R04, 11R06, $11 R 33$.

Keywords: Mahler measure, Pisot numbers, free groups. 
every Perron number is an algebraic integer, so $\mathcal{M}^{*} \subset P$. Furthermore, Boyd [3] showed that not every Perron integer belongs to $\mathcal{M}^{*}$. For instance, if $\alpha$ is the largest positive root of $X^{d}-X-1=0, d \geqslant 4$, then $\alpha$ is a Perron number, but $\alpha \notin \mathcal{M}^{*}$. (To be precise, Boyd proved that $\alpha \notin \mathcal{M}$, but his argument easily extends to the following criterion: if $\alpha \in \mathcal{M}^{*}$ then its conjugates different from $\alpha$ all have moduli smaller than $\alpha$ and greater than or equal to $\alpha^{-1}$.) In [6] the author showed that, for every Perron number $\alpha$, there is a positive integer $n$ such that $n \alpha \in \mathcal{M}$, so $\alpha$ can be written as a quotient of two Mahler measures $n \alpha$ and $n=M(n)$. On the other hand, say $\sqrt{2}=M(\sqrt{2}+2) / M(\sqrt{2}+1)$ is not a Perron number, hence $P \subset G$. The final relation between the sets $G$ and $\overline{\mathbb{Q}}_{>0}$ is an unexpected one: it turns out that $G=\overline{\mathbb{Q}}_{>0}$. Summarizing, we have that

$$
\mathcal{M} \subset \mathcal{M}^{*} \subset P \subset G=\overline{\mathbb{Q}}_{>0} .
$$

We will prove the equality $G=\overline{\mathbb{Q}}_{>0}$ in a more special and precise form. Recall that $\alpha>1$ is a Pisot number if it is an algebraic integer whose other conjugates (if any) all lie strictly inside the unit circle. We call $\alpha>1$ a generalized Pisot number if it satisfies the above definition but without assumption that $\alpha$ is an algebraic integer. (This definition follows the line proposed in [7].) Of course, every generalized Pisot number is a Perron number and every Perron number is a certain rational multiple of a generalized Pisot number.

We are now able to give our first result which implies that the free group under multiplication $G$ generated by $\mathcal{M}$ is the multiplicative group of all positive algebraic numbers.

Theorem 1. For every positive algebraic number $\alpha$ there exist two generalized Pisot numbers $\beta, \gamma \in \mathbb{Q}(\alpha)$ such that $\alpha=M(\beta) / M(\gamma)$.

What happens if we replace multiplication by addition? Although now the multiplicative structure of the Mahler measure will be mixed with the additive structure of a group one may already expect that the situation will be similar to that above. This is indeed the case. Let $\mathcal{M}^{+}$be a monoid under addition generated by $\mathcal{M}$, and let $A=\left\{\sum k_{j} m_{j} \mid m_{j} \in \mathcal{M}, k_{j} \in \mathbb{Z}\right\}$ be a free additive group generated by $\mathcal{M}$. We begin with an example showing that $\mathcal{M} \neq \mathcal{M}^{+}$.

Theorem 2. Let $d \geqslant 4$ be an even integer. Then $\alpha>1$ solving $X^{d}-X^{d-1}-$ $\cdots-X-1=0$ is a Pisot number, and $\alpha+1 \in \mathcal{M}^{+} \backslash \mathcal{M}$.

Boyd's example of the positive root $\alpha$ of $X^{d}-X-1=0$ also shows that $\mathcal{M}^{+} \neq P$, since every $\alpha<2$ lying in $\mathcal{M}^{+}$must belong to $\mathcal{M}$. Note that $\sqrt{2}=$ $M(2 \sqrt{2}+2)-M(\sqrt{2}+2)$ is not a Perron number, so $P \neq A$. Hence, in the additive case, we have that

$$
\mathcal{M} \subset \mathcal{M}^{+} \subset P_{0} \subset A,
$$

where $P_{0}$ stands for the set of Perron numbers which are algebraic integers. Clearly, every element of $A$ is a real algebraic integer. As above, the converse is also true. Once again we give it in a more precise form. 
Theorem 3. For every real algebraic integer $\alpha$ there exist four generalized Pisot numbers $\beta, \gamma, \beta^{\prime}, \gamma^{\prime} \in \mathbb{Q}(\alpha)$ and two positive integers $b, c$ such that $\alpha=$ $b M(\beta)+c M(\gamma)-b M\left(\beta^{\prime}\right)-c M\left(\gamma^{\prime}\right)$.

The reason that four numbers appear instead of two is that in the proof below we first express a real algebraic integer as a difference of two Perron integers and then each of these two as a linear form in two Mahler measures. In conclusion, we ask whether, similarly to the multiplicative case, every real algebraic integer can be expressed as a difference of two Mahler measures.

\section{Main lemma}

We will first show that there is a Tschirnhausen transformation which maps a real algebraic number $\sigma$ into another real algebraic number in the field $\mathbb{Q}(\sigma)$ which is large compared to its other conjugates.

Lemma. Let $\sigma$ be a fixed real algebraic number of degree $d \geqslant 2$ over $\mathbb{Q}$ with its other conjugates $\sigma_{2}, \ldots, \sigma_{d}$. Then there is a positive number $\lambda=\lambda(\sigma)$ depending on $\sigma$ only such that, for every sufficiently large number $T$, there is a polynomial $f(X) \in \mathbb{Z}[X]$ (depending on $T$ ) for which $f(\sigma)>T$ and $\max _{2 \leqslant j \leqslant d}\left|f\left(\sigma_{j}\right)\right|<\lambda T^{(d-2) /(d-1)}$.

Proof. Set $\ell_{j}=\prod_{i}\left|\sigma_{i}-\sigma_{j}\right|$, where the product is taken over every $i \neq j$ in the range $2 \leqslant i \leqslant d$, and $\ell=\prod_{j=2}^{d}\left|\sigma-\sigma_{j}\right|$. Also, putting $\sigma_{1}=\sigma$, let $\operatorname{sep}(\sigma)=\min _{1 \leqslant i<j \leqslant d}\left|\sigma_{i}-\sigma_{j}\right|$.

Let $L$ be a large positive integer to be chosen later, and let $n_{j}$ be the Gaussian integer $\mathbb{Z}+\mathbb{Z} \sqrt{-1}$ nearest to $L \sigma_{j}$. (In case if there are several of these, let $n_{j}$ be the smallest in absolute value.) Consider the polynomial $f_{1}(X)=\prod_{j=2}^{d}(L X-$ $\left.n_{j}\right)$. Evidently, $n_{j} \in \mathbb{Z}$ for every $j$ with real $\sigma_{j}$. Also $\left(L X-n_{j}\right)\left(L X-\bar{n}_{j}\right) \in \mathbb{Z}[X]$. It follows that $f_{1}(X) \in \mathbb{Z}[X]$, since all complex conjugates of $\sigma$ come in complex conjugate pairs.

By the choice of $n_{j},\left|L \sigma_{j}-n_{j}\right| \leqslant 1 / \sqrt{2}$. From $\left|L \sigma_{i}-n_{j}\right| \leqslant\left|L\left(\sigma_{i}-\sigma_{j}\right)\right|+$ $\left|L \sigma_{j}-n_{j}\right|$ it follows that $\left|L \sigma_{i}-n_{j}\right|$ is at most $2 L\left|\sigma_{i}-\sigma_{j}\right|$ for $i \neq j$ (provided that $L>1 / \operatorname{sep}(\sigma))$. Hence, the upper bound $\left|f_{1}\left(\sigma_{j}\right)\right|<(2 L)^{d-2} \ell_{j}$ holds for every $j=2, \ldots, d$. Similarly, since $\left|L \sigma-n_{j}\right| \geqslant\left|L\left(\sigma-\sigma_{j}\right)\right|-\left|L \sigma_{j}-n_{j}\right|>(L / 2)\left|\sigma-\sigma_{j}\right|$ for $L>\sqrt{2} / \operatorname{sep}(\sigma)$, we see that $\left|f_{1}(\sigma)\right|>(L / 2)^{d-1} \ell$. In order to complete the proof it is sufficient to take $f(X)=f_{1}(X)^{2}, L=\left[2\left(T / \ell^{2}\right)^{1 / 2(d-1)}\right]+1$ and $\lambda=36 \ell^{-2 /(d-1)} \max _{2 \leqslant j \leqslant d} \ell_{j}^{2}$.

\section{Proofs}

Proof of Theorem 1. Let $\alpha$ be a positive algebraic number, and let $t$ be a positive integer such that $t \alpha$ is an algebraic integer. We apply the lemma for $\sigma=$ $t \alpha$. Assume that $f(X)$ is the resulting polynomial. Then both $\xi=t \alpha f(t \alpha)$ and $\zeta=t f(t \alpha)$ are algebraic integers. For $T$ sufficiently large, they are both of degree $d$. Choose two primes $p$ and $q$ which are so large that $p, q>\max \{H(\xi), H(\zeta)\}$, 
where $H$ stands for the height of an algebraic number, i.e. the largest modulus of the coefficients of its minimal polynomial, and satisfy

$$
\lambda t \max _{2 \leqslant j \leqslant d}\left\{1,\left|\alpha_{j}\right|\right\} T^{(d-2) /(d-1)}<p / q<T /(t \max \{1, \alpha\}) .
$$

Then $\beta=q \xi / p$ and $\gamma=q \zeta / p$ are generalized Pisot numbers lying in $\mathbb{Q}(\alpha)$. Moreover, the leading coefficients of their minimal polynomials are both equal to $p^{d}$. Thus $M(\beta)=p^{d-1} q \xi=p^{d-1} q t \alpha f(t \alpha)$ and $M(\gamma)=p^{d-1} q \zeta=p^{d-1} q t f(t \alpha)=$ $M(\beta) / \alpha$, giving the result for every $\alpha$ of degree $d \geqslant 2$. For rational $\alpha=u / v$, where $u$ and $v$ are positive integers, we simply have $\alpha=M(u) / M(v)$.

Proof of Theorem 2. The fact that $X^{d}-X^{d-1}-\cdots-X-1=0, d \geqslant 2$, defines a Pisot number $\alpha$, where $1<\alpha<2$, is well-known (see, e.g., [5], [9]) and follows easily from Rouché's theorem. Thus $\beta=\alpha+1 \in \mathcal{M}^{+}$, since $\alpha=M(\alpha) \in \mathcal{M}$ and $1=M(1) \in \mathcal{M}$. The number $\beta<3$ is the largest positive root of its minimal polynomial $g(X)=(X-1)^{d}-\left((X-1)^{d}-1\right) /(X-2) \in \mathbb{Z}[X]$. If $\beta \in \mathcal{M}^{*}$ then, by Boyd's condition mentioned above, we would have that all conjugates of $\beta$ over $\mathbb{Q}$ are greater than $1 / 3$ in absolute value. However, for even $d \geqslant 4$, we have that $g(0)=1>0$ and $g(1 / 3)=\left(8(-2 / 3)^{d}-3\right) / 5 \leqslant-23 / 81<0$. So $g(X)$ has a root in the interval $(0,1 / 3)$, a contradiction. This proves that $\beta \in \mathcal{M}^{+} \backslash \mathcal{M}^{*}$ which is more than required.

Proof of Theorem 3. We now apply the lemma for $\sigma=\alpha$, and assume that the resulting polynomial is $f(X)$. Then $\xi=f(\alpha)$ and $\zeta=\alpha+f(\alpha)$ are real algebraic integers. For $T$ is sufficiently large, they are both of degree $d$. (Moreover, they are both Perron integers.) Let us take four distinct prime numbers $p, q, p^{\prime}, q^{\prime}>\max \{H(\xi), H(\zeta)\}$ such that the quotients $p / q$ and $p^{\prime} / q^{\prime}$ both lie between $2 \max \left\{\lambda T^{(d-2) /(d-1)}, \max _{2 \leqslant j \leqslant d}\left|\alpha_{j}\right|\right\}$ and $T-|\alpha|$. Then $\beta=q \zeta / p, \beta^{\prime}=$ $q \xi / p, \gamma=q^{\prime} \xi / p^{\prime}$ and $\gamma^{\prime}=q^{\prime} \zeta / p^{\prime}$ are generalized Pisot numbers lying in $\mathbb{Q}(\alpha)$. Furthermore, the leading coefficients of the minimal polynomials of $\beta, \beta^{\prime}, \gamma$ and $\gamma^{\prime}$ are, respectively, $p^{d}, p^{d}, p^{\prime d}$ and ${p^{\prime}}^{d}$. Thus $M(\beta)=p^{d-1} q \zeta, M\left(\beta^{\prime}\right)=p^{d-1} q \xi$, $M(\gamma)={p^{\prime}}^{d-1} q^{\prime} \xi$ and $M\left(\gamma^{\prime}\right)={p^{\prime}}^{d-1} q^{\prime} \zeta$. Choosing positive integers $b$ and $c$ which satisfy $b p^{d-1} q-c p^{\prime-1} q^{\prime}=1$, we deduce that $\zeta=b M(\beta)-c M\left(\gamma^{\prime}\right)$ and $\xi=b M\left(\beta^{\prime}\right)-c M(\gamma)$. Hence $\alpha=\zeta-\xi=b M(\beta)+c M(\gamma)-b M\left(\beta^{\prime}\right)-c M\left(\gamma^{\prime}\right)$. This proves the theorem for every algebraic integer $\alpha$ of degree $d \geqslant 2$. For $\alpha=k \in \mathbb{Z}$, we trivially have $k=M(|k|+k+1)+M(1)-M(|k|+1)-M(1)$.

\section{Acknowledgements}

The author wishes to thank the Department of Mathematics at Frankfurt am Main University, where the paper was written in June 2003, for the invita-

tion. The research was partially supported by the Lithuanian State Science and Studies Foundation.

\section{References}

[1] R.L. Adler and B. Marcus, Topological entropy and equivalence of dynamical systems, Mem. Amer. Math. Soc. 20 (1979). 
[2] D.W. Boyd, Speculations concerning the range of the Mahler measure, Canad. Math. Bull. 24 (1981), 453-469.

[3] _ Perron units which are not Mahler measures, Ergod. Th. and Dynam. Sys. 6 (1986), 485-488.

[4] J.D. Dixon and A. Dubickas, The values of Mahler measures, Mathematika (to appear).

[5] A. Dubickas, Mahler measures close to an integer, Canad. Math. Bull. 45 (2002), 196203.

[6] - On numbers which are Mahler measures, Monatsh. Math. 141 (2004), 119-126.

[7] A. Dubickas and C.J. Smyth, On the Remak height, the Mahler measure, and conjugate sets of algebraic numbers lying on two circles, Proc. Edinburgh Math. Soc. 44 (2001), $1-17$.

[8] D.H. Lehmer, Factorization of certain cyclotomic functions, Ann. of Math. (2) 34 (1933), 461-479.

[9] J.F. Traub, Iterative methods for the solution of equations, 2nd ed., Chelsea, New York, 1982.

Department of Mathematics and Informatics Vilnius University, Naugarduko 24, 03225 Vilnius, LithuANiA

E-mail address: arturas.dubickas@maf .vu.It 